Álvarez Botello, Julio; Torres Velázquez, Alejandra Magali; Chaparro Salinas, Eva Martha (2016). Diagnóstico del liderazgo educativo en las Instituciones de Educación Superior del Valle de Toluca. Revista de Investigación Educativa, 34(1), 51-68. DOI: http://dx.doi.org/10.6018/rie.34.1.206881

\title{
Diagnóstico del liderazgo educativo en las Instituciones de Educación Superior del Valle de Toluca
}

\author{
Diagnosis of educational leadership \\ in higher Education Institutions Valley Toluca
}

Julio Álvarez Botello*,Alejandra Magali Torres Velázquez**, Eva Martha Chaparro Salinas***

\begin{abstract}
Resumen
En educación el liderazgo alude a la gestión adecuada y efectiva del personal docente, de tal forma que lleve a las instituciones educativas a ser competitivas y de calidad. De lo anterior, surge la presente investigación de corte cuantitativa, descriptiva y transversal, cuyo objetivo general es diagnosticar si el Modelo de Liderazgo Educativo es practicado en las Instituciones de Educación Superior del Valle de Toluca, siendo los objetivos específicos identificar las variables que componen tal modelo: Liderazgo Pedagógico, Liderazgo Directivo y Liderazgo Institucional, respondiendo así a las preguntas de investigación planteadas a través de la recopilación de información por medio de un instrumento cuya escala de medición fue tipo Likert, aplicado a una muestra representativa de 387 docentes de las IES del Valle de Toluca; en donde los resultados generales arrojaron que el Modelo de Liderazgo Educativo, no es practicado en las instituciones estudiadas, abriendo la pauta para futuras intervenciones.

Palabras clave: Liderazgo, Educación; Docentes; Instituciones de educación superior.

* Doctor en Ciencias de la Educación. Profesor de tiempo completo de la Universidad Autónoma del Estado de México (UAEMex). Investigador perteneciente al cuerpo académico en Administración de Organizaciones Educativas y Presidente de la Red de Investigación Latinoamericana en Competitividad Organizacional. E-mail: caaoe@hotmail.com

** Licenciada en Psicología por la Facultad de Ciencias de la Conducta de la UAEMex. Maestrante del $4^{\circ}$ Semestre de la Maestría en Administración de Recursos Humanos por la Facultad de Contaduría y Administración de la UAEMex. E-mail: alejandramagali_tv@hotmail.com

*** Doctora en Ciencias de la Educación. Profesor de tiempo completo de la UAEMex. Investigadora perteneciente al cuerpo académico en Administración de Organizaciones Educativas e integrante de la Red de Investigación Latinoamericana en competitividad organizacional. E-mail: bebachaparro@yahoo.com.mx
\end{abstract}




\begin{abstract}
Leadership in education refers to the proper and effective management of teachers, so that it leads educational institutions to be competitive and to have quality. From the above, results the present investigation of a quantitative, descriptive and transversal nature, which overall objective is to diagnose whether the Model of Educational Leadership is practiced in Institutions of Higher Education of the Toluca Valley, with the specific objectives to identify the variables that make up such Model: Educational Leadership, Executive Leadership and Institutional Leadership, responding to the research questions through the collection of information by means of an instrument using the Likert measurement scale; it was applied to a representative sample of 387 teachers from IES Toluca Valley, where the overall results showed that the model of Educational Leadership is not practiced in the institutions studied, opening the tone for future interventions.
\end{abstract}

Keywords: Leadership, Education, Teachers, Institutions of higher education.

\title{
Introducción
}

Derivado de los diferentes y acelerados acontecimientos mundiales de trascendencia histórica que trastocan el quehacer humano, las instituciones educativas han tenido que irse adaptando y transformado a las exigencias de los cambios derivados de tal dinamismo. Cambios políticos, económicos, medio ambientales, sociales y culturales han tenido un impacto significativo en la educación latina. Garbanzo y Orozco (2010), refieren que estos cambios implican, entre otras cosas, la rapidez con la que los conocimientos y prácticas, asumidas como válidas en los distintos ámbitos de la actividad humana, quedan obsoletas y, como consecuencia, los efectos no son otros que buscar nuevas pautas de desarrollo y competitividad para las organizaciones educativas.

Hablar de liderazgo y educación, es hablar de cambio, dinamismo, competitividad, calidad, sociedad, país, desarrollo y crecimiento; en la actualidad ya no es válido hablar de directores escolares, ahora el concepto ha cambiado por líder educativo o director - líder escolar, ya que éstos son los encargados de coadyuvar al crecimiento educativo de un país; labor que si bien es compleja, se hace aún más al hablar de Instituciones de Educación Superior, en donde el nivel de exigencia hacia los dirigentes se encamina a la satisfacción del personal que tienen a su cargo y a la satisfacción de las necesidades profesionales de los alumnos.

“En el ámbito educativo, el ejercicio del liderazgo está matizado por la responsabilidad del directivo - docente en la promoción de cambios que desprenden de la interacción del grupo de alumnos y alumnas, padres, colegas y otros miembros de la comunidad educativa" (González González y González Cubillán, 2012).

Como líder en la educación superior, el director académico tiene variedad de responsabilidades y muchos retos que enfrentar, que tienden a evolucionar con la siempre cambiante cara de la educación superior, por lo que su papel es multifacético y a veces ambiguo (Elizondo Montemayor, 2011). Parra (citado por González González y González Cubillán, 2012), define al líder, dentro de la educación, como aquella persona con capacidad de optimizar el uso de las técnicas y métodos para el trabajo pedagógico en beneficio del mejoramiento y de la calidad educativa. De ello, la presente 
investigación concibe al liderazgo como un elemento indispensable para el logro de la competitividad educativa.

Liderazgo, concepto retomado de las ciencias administrativas y adaptado a múltiples ámbitos del quehacer humano hace presencia en el sector educativo para enfatizar la necesidad de dirigentes que cuenten con las características necesarias que lleven a una institución educativa a ser eficaz, eficiente y sobretodo efectiva, para crecer y desarrollarse en un ambiente altamente dinámico en donde los conocimientos cambian, se ajustan o adaptan a la nueva información que viaja rápidamente.

\section{Revisión teórica}

El liderazgo, como fenómeno universal, parte del instinto gregario del ser humano, de la necesidad de agruparse, organizarse y conducir sus actividades profesionales, familiares y sociales en beneficio de una convivencia que garantice una mejor calidad de vida.

Lussier y Achua (2010), refieren que el tema de liderazgo ha generado emoción e interés desde la antigüedad. Cuando se piensa en el término: liderazgo, se asocia casi inmediatamente con imágenes de individuos dinámicos y poderosos quienes dirigen ejércitos victoriosos, modelan sucesos de las naciones, crean religiones o dirigen imperios corporativos; sin embargo, mencionan los autores, no existe una definición universal del liderazgo porque éste es complejo y se analiza en distintas formas, las cuales requieren de diversas definiciones; no obstante plantean su concepto de liderazgo refiriéndose a él como: "El liderazgo es el proceso de influencia entre líderes y seguidores para logar los objetivos organizacionales por medio del cambio" (p. 6).

En el ámbito educativo, el liderazgo es un elemento sine qua non que lleva a la calidad y competitividad escolar ya queexiste una tendencia mundial a reformar la educación, motivada por la necesidad que tienen las universidades para reflejar los requerimientos futuros de la sociedad y para asegurar que los estudiantes adquieran aptitudes apropiadas para ejercer su profesión con calidad (Boelen, 1993, citado por Elizondo Montemayor, 2011). A este respecto, Cheong Cheng (2011), refiere que los líderes escolares al ser más estratégicos en su liderazgo y liderar sus escuelas de forma proactiva y competitivase deben enfrentar a los desafíos contextuales con estrategias adecuadas.

Complementando lo anterior, Álvarez Botello et al. (2012), mencionan que el liderazgo educativo tiene que tener las siguientes características:

- Debe ser compartido y distribuido.

- La dirección debe ser centrada en el desarrollo de las personas tanto individual como colectivamente.

- Debe tener una dirección visionaria.

- Debe asumir riesgos.

- Debe estar directamente implicado en las decisiones pedagógicas.

- La dirección debe estar formado en proceso de cambio.

Así, en función a lo anterior, se retoma el modelo de Liderazgo Educativo expuesto por Abad (2010), en su libro: 7 ideas clave. Escuelas sostenibles en convivencia, para el desarrollo de la presente investigación, el cual se expone a continuación. 


\section{Modelo de liderazgo educativo}

\section{Liderazgo Institucional}

Las administraciones educativas tienen la responsabilidad de crear las mejores condiciones para que los centros sean lugares donde todos los alumnos convivan, aprendan y tengan éxito en sus objetivos académicos. La realidad muestra que en muchas ocasiones existe una desconexión entre los intereses y necesidades de la administración y los del profesorado, alumnado y familias (Abad, 2010).

El liderazgo institucional pasa por conectar las exigencias sociales con las necesidades de los docentes y alumnos. En temas relacionados con la convivencia, este liderazgo se concreta básicamente en lo siguiente:

- Ofrecer un marco legal para poder hacer de la gestión de la convivencia un objetivo de aprendizaje democrático.

- Impulsar la formación del profesorado en la gestión de la convivencia en el centro y en el aula.

- Promover y apoyar el funcionamiento de estructuras participativas de profesorados y alumnos en el centro, con su correspondiente reconocimiento en el horario.

- Evaluar y ofrecer apoyo y reconocimiento a los centros cuando realicen prácticas eficaces.

\section{Liderazgo Directivo}

Continuando con Abad (2010), menciona que en este tipo de liderazgo educativo, los equipos directivos de cada centro juegan un papel clave en la definición del modelo de la convivencia escolar. De forma implícita o explícita todos los equipos directivos toman decisiones que afectan a la manera de detectar, intervenir y prevenir los conflictos derivados de la convivencia diaria.

A diferencia de lo expuesto por Abad (2010), SummersDonna (2006), refiere que el estilo de liderazgo directivo es autocrático. Por lo general, comenta, se utiliza cuando el líder debe tomar decisiones unilaterales que serán seguidas sin comentarios ni cuestionamientos de los seguidores. La necesidad de emplear este estilo de liderazgo puede ser resultado de que el líder tenga más conocimiento de la situación, o de que la decisión afectará el bien común de la organización. Este tipo de liderazgo puede identificarse por determinaciones tan simples como "no se tolerarán juegos entre los empleados durante el trabajo".

\section{Liderazgo Pedagógico}

La implantación de un modelo de gestión de calidad dentro de las organizaciones educativas necesita un impulso institucional y el estímulo y la determinación del equipo directivo, pero también exige un liderazgo capaz de ofrecer seguridad sobre el terreno que pisa, así como gestionar los procesos de participación. A este tipo de lide- 
razgo Abad (2010), lo denomina liderazgo pedagógico y su principal objetivo es facilitar la creación de redes de trabajo orientadas a la mejora de la convivencia. El liderazgo pedagógicopromueve, por tanto, la participación de la comunidad educativa en la toma de decisiones y la responsabilidad en la puesta en marcha de las medidas de mejora. Se trata de un verdadero liderazgo compartido.

A diferencia de los liderazgos institucional y directivo, el autor refiere que en el caso del liderazgo pedagógico no queda inicialmente definido sobre qué estructura o personas debe recaer dicha función. Desarrollar el liderazgo pedagógico en un proyecto de gestión de calidad exige tanto el deseo de ejercer dicho liderazgo como la posibilidad de llevarlo a cabo.

Otra característica, del liderazgo pedagógico, en la que el autor enfatiza está relacionada con la capacidad para facilitar procesos grupales. Gestionar la convivencia de forma participativa exige crear equipos de trabajo que compartan responsabilidades y que estén liderados.

Un equipo de trabajo formado por profesorado del centro que trabaja de forma coordinada y con un alto grado de reconocimiento por parte de sus compañeros es capaz de trasladar esa dinámica de trabajo a otros grupos de alumnos con los que posteriormente va a trabajar. Esta manera de ejercer el liderazgo refleja un estilo de trabajo con grupos denominado por Abad (2010), como superliderazgo.

Como se puede observar, el liderazgo pedagógico se vuelca sobre el trabajo grupal y destina una parte importante de su energía a crear estructuras participadas por el alumnado y profesorado. Cuando esto no es así, la persona que ejerce el liderazgo acaba asumiendo demasiado trabajo y cansándose de ser un referente solitario para sus compañeros y para el alumnado.

\section{Preguntas de investigación}

Garbanzo y Orozco (2010), mencionan que las organizaciones educativas son los espacios en donde se concreta el proceso de enseñanza - aprendizaje y que además de desarrollar las capacidades cognitivas de los estudiantes también potencializan su formación integral, no sólo a través del conocimiento, también por medio de la ética, estética, moral y valores. Dada toda esta complejidad, el liderazgo mediante el cual se gestan las organizaciones educativas debe trascender de un corte tradicional a un liderazgo renovador, sobre todo en las instituciones de Educación Superior en donde la formación debe manifestarse en el ámbito humano, es decir, en la cultura y por ende en la sociedad.

De lo anterior, surge la investigación, "Diagnostico del liderazgo educativo en las Instituciones de Educación Superior del Valle de Toluca", trabajo con el que se diagnosticó de forma cuantitativa la práctica del liderazgo educativo en las Instituciones Educativas de Nivel Superior; de lo anterior, las preguntas de investigación que se generaron fueron:

1) ¿El liderazgo directivo es practicado en las Instituciones de Educación Superior del Valle de Toluca?

2) ¿Qué tan practicado es el liderazgo institucional en las Instituciones de Educación Superior del Valle de Toluca? 
3) ¿El liderazgo pedagógico es practicado en las Instituciones de Educación Superior del Estado de México?

4) ¿Existen diferencias estadísticamente significativas en la práctica del liderazgo educativo en los organismos estudiados?

\section{Objetivos de la investigación}

\section{Objetivo general:}

Diagnosticar el liderazgo educativo en las Instituciones de Educación Superior del Valle de Toluca.

\section{Objetivos específicos:}

- Determinar qué tan practicado es el liderazgo directivo en las Instituciones de Educación Superior en el Valle de Toluca.

- Determinar qué tan practicado es el liderazgo institucional en las Instituciones de Educación Superior del Valle de Toluca.

- Determinar qué tan practicado es el liderazgo pedagógico en las Instituciones de Educación Superior del Valle de Toluca.

- Determinar, si existen diferencias estadísticamente significativas en la práctica del liderazgo educativo en los organismos estudiados.

\section{Revisión de investigaciones similares}

En los últimos años se han ido desarrollando múltiples investigaciones en lo que respecta a la calidad y competitividad educativa, relacionando variables como: liderazgo, dirección de personal, mejora continua, etcétera. En relación a la temática de la investigación se encuentra la investigación realizada por Sánchez, Tapia y Becerra (2006), "Liderazgo: una mirada desde directivos, profesores y alumnos", cuyo objetivo fue conocer los diferentes significados que los alumnos, profesores y directores de escuelas chilenas atribuyen al concepto: liderazgo directivo.

Asimismo, López Leiva (2010), con su estudio "Liderazgo y mejora educativa", considera al liderazgo y a la calidad educativa como la complejidad del sistema educativo chileno, brindando un panorama bibliográfico sobre los puntos trascendentales del tema y refiriendo que para mejorar la educación, en cualquier nivel, es necesario que existan directivos de calidad que afronten los nuevos retos del sistema escolar.

Otro trabajo desarrollado fue el denominado: "Liderazgo de los directivos docentes en contextos vulnerables", investigación que se realizó en Colombia y cuyo propósito fue describir el liderazgo directivo en establecimientos educacionales de enseñanza media en contextos vulnerables (Tapia, Mansilla-Sepúlveda, Becerra y Saavedra, 2011).

Rodríguez-Molina (2011), plantea la problemática de conocer quiénes son los líderes pedagógicos, sus procedimientos de supervisión e instancias de retroalimentación y autoevaluación, a través del proyecto de investigación denominado "Funciones y rasgos del liderazgo pedagógico en los centros de enseñanza", cuyos hallazgos principales muestran una diferencia explícita entre el líder administrativo y el pedagógico, en donde este último es valorado positivamente por el cuerpo docente. 
Por su parte, González González y González Cubillán (2012), presentan el trabajo "Estilos de Liderazgo en el docente universitario", cuyo objetivo es la descripción de las características de los estilos de liderazgo laissez faire, autocrático, transaccional y transformacional presentes en un grupo de profesores y profesoras de la Facultad de Arquitectura y Diseño de la Universidad del Zulia, Venezuela. Trabajo en el que los resultados obtenidos apuntan hacia la tendencia a adoptar comportamientos característicos de un liderazgo transformacional bajo las siguientes combinaciones: autocrático- transformacional, transaccional-transformacional, laissez faire-autocráticotransformacional y autocrático-transaccional-transformacional. Los datos obtenidos señalan que los y las docentes muestran preocupación por la promoción de sus estudiantes a través de la atención a sus necesidades de logro, de estimulación intelectual y de crecimiento personal, apoyando su prosecución estudiantil en medio de un clima académico favorable al desarrollo personal y del grupo.

De igual forma, González González (2008), es su investigación “Liderazgo transformacional en el docente universitario" tuvo por objetivo determinar el liderazgo predominante en los docentes de la Facultad de Arquitectura y Diseño de la Universidad de Ziula, Venezuela.

Adan (2006), con su propuesta investigativa denominada "Liderazgo renovador y esperanzador para construir la universidad del futuro" pretende desarrollar una propuesta de liderazgo educativo universitario denominado Liderazgo esperanzador y renovador, esto para construir universidades que atiendan las necesidades sociales futuras a través de la integración y cooperación de todos los actores universitarios.

Finalmente, Ahumada (2012), refiere que las investigaciones que han intentado abordar el tema de liderazgo en los contextos educativos en los últimos años, han surgido como una necesidad de dar respuesta al fracaso en la calidad y competitividad educativa, sobre todo en países subdesarrollados, como lo son la mayoría de las naciones latinas. En Estados Unidos, por ejemplo, se resalta la importancia del desarrollo de un modelo de liderazgo centrado en "aprender a aprender" y en el "compartir prácticas pedagógicas". En Reino Unido también se ha fortalecido la idea de un "liderazgo para el aprendizaje", sin embargo, tanto en Inglaterra como en Escocia existe una crisis de este modelo dado que los profesores no quieren asumir roles de dirección. En Latinoamérica, ha ido evolucionando la educación que ha pasado de mirar al director como un administrador y ejecutor de las políticas emanadas del nivel central, a un director-líder en la transformación y en los procesos de cambio que se viven en la escuela.

\section{Metodología}

\section{Alcances de la investigación}

El liderazgo es un factor importante y relevante para el sector educativo de Nivel Superior, ya que con este se fomenta la competitiva educativa para todos los involucrados en la gestión del sistema escolar. Sin embargo, aún con la gran cantidad de información existente, en estos días no se conoce una perfil de liderazgo educativo en las Instituciones de Educación Superior del Valle de Toluca, es decir, en este sector de la población es un tema poco estudiado e indagado. 
De lo anterior la proposición de investigación "Diagnostico del liderazgo educativo en las Instituciones de Educación Superior del Valle de Toluca", tiene un tipo de alcance Cuantitativo y Descriptivo ya que indaga un problema poco estudiado para la población a la que se dirige y lo averigua desde una perspectiva innovadora, ayudando a identificar conceptos promisorios y preparando el campo para nuevos estudios relacionados. Asimismo, mide de forma cuantitativa los conceptos relacionados con liderazgo y define claramente sus variables.

\section{Tipo de investigación}

El diseño de investigación del proyecto: "Diagnostico del liderazgo educativo en las Instituciones de Educación Superior del Valle de Toluca", es de tipo Transeccional o Transversal explicativo-descriptivo, ya que de acuerdo con Hernández Sampieri, Fernández Collado y Baptista Lucio (2010), tienen como objetivo indagar la incidencia de las modalidades o niveles de una variable en una población determinada (Valle de Toluca) en un tiempo determinado, es decir se recopilarán datos en un momento único para una población específica.

\section{Variables de investigación}

Variable dependiente:

- Liderazgo Educativo

Variables independientes:

- Liderazgo institucional

- Liderazgo directivo

- Liderazgo pedagógico

\section{Definición Operacional de las variables:}

La variable dependiente: liderazgo se medió a través del cuestionario: “Liderazgo educativo" validado y confiable, para la presente investigación.

\section{Población y muestra de la investigación}

Población o Universo: Docentes de tiempo completo, medio tiempo y de horas clase de las Instituciones de Educación Superior del Valle de Toluca.

De lo anterior y de acuerdo a la Asociación Nacional de Universidades e Instituciones de Educación Superior (ANIUES, 2012), la población total a estudiar fue de 10,289 profesores de tiempo completo, medio tiempo y de horas clase para el sector que comprende las instituciones, tanto públicas como privadas de los municipios de:

- Almoloya de Juárez

- Calimaya

- Lerma
- Metepec

- Ocoyoacac

- Otzolotepec 
- San Mateo Atenco

- Santiago Tianguistenco

- Temoaya
- Tenango del Valle

- Toluca

- Zinacantepec

Tal muestra sirvió para medir las tres variables que componen el estudio: Liderazgo Institucional, Liderazgo Directivo y Liderazgo Pedagógico, dado que el instrumento utilizado condensaba las tres variables.

Muestra: En virtud de lo expuesto la muestra con la que se trabajó fue obtenida estadísticamente, por medio del programa STATS, utilizando un margen de error del $5 \%$, un nivel de confianza del $95 \%$ y un nivel de heterogeneidad del $50 \%$ obteniendo así que la muestra representativa fue $\mathbf{3 7 1}$ profesores. De acuerdo a la representatividad escolar de cada municipio, la aplicación debería haberse estratificado tal como se muestra en la Tabla 1:

Tabla 1

Representatividad de la muestra de docentes por municipio

\begin{tabular}{|c|c|c|c|c|c|c|}
\hline \multirow[b]{2}{*}{ Profesores } & \multicolumn{6}{|c|}{ Cantidad de cuestionarios a aplicarse } \\
\hline & Toluca & Metepec & Tianguistenco & Otros & SUBTOTAL & TOTAL \\
\hline $\begin{array}{l}\text { Profesores de tiempo } \\
\text { completo y medio tiem- } \\
\text { po de escuelas públicas. }\end{array}$ & 70 & 25 & 14 & 4 & 113 & 257 \\
\hline $\begin{array}{l}\text { Profesores de horas clase } \\
\text { de escuelas públicas. }\end{array}$ & 80 & 28 & 20 & 16 & 144 & \\
\hline $\begin{array}{l}\text { Profesores de horas clase } \\
\text { de escuelas privadas } \mathrm{PH}\end{array}$ & 42 & 29 & 4 & 19 & 94 & \\
\hline $\begin{array}{l}\text { Profesores de tiempo } \\
\text { completo y medio tiem- } \\
\text { po de escuelas privadas. }\end{array}$ & 12 & 6 & 1 & 1 & 20 & 114 \\
\hline TOTAL & & & & & & 371 \\
\hline
\end{tabular}

(Elaboración propia, 2014).

Sin embargo, dado el alcance de la muestra, en la aplicación se encuestaron a 291 profesores de escuelas públicas y a 96 en escuelas privadas, para los tres tipos de liderazgo analizados, dando un total de $\mathbf{3 8 7}$ instrumentos aplicados lo que supero la muestra en 16 observaciones.

\section{Diseño del instrumento}

Para fines de la presente investigación, se diseñó el instrumento "Liderazgo educativo". En la elaboración del mismo, diseñado para docentes de Instituciones de Educación Superior, se tomó como referencia el modelo de liderazgo educativo expuesto por Abad (2010), quien menciona que el Liderazgo Educativo debe ir 
enfocado a tres variables: Liderazgo Institucional, Liderazgo Directivo y Liderazgo Pedagógico; por lo que en virtud de esta clasificación se elaboraron 23 reactivos auto administrados tipo Likert, con lo que se dio forma al cuestionario para su validación y confiabilidad.

En referencia a la validez del mismo, ésta se obtuvo por medio del Análisis Factorial Exploratorio, a través del Software Estadístico SPSS 20.1, con una muestra representativa de 201 observaciones, ya que de acuerdo con Morales Vallejo (2013) para que una orientación sea segura en un Análisis Factorial, es recomendable que el número de observaciones o de sujetos no sea menor de 200 y que al menos haya 5 sujetos por ítem. Complementando lo anterior, Martín Martín, Cabero Moran y De paz Santana (2008), mencionan que para realizar este análisis es regla general que existan el mayor número de observaciones posibles o bien 4 o 5 por reactivo. De los resultados obtenidos del Análisis Factorial, se obtiene que:

1. La Medida de Adecuación Muestral de Kaiser - Meyer - Olkin es de .938 con una significancia de $\mathbf{. 0 0 0}$, lo que refiere que es un instrumento válido para ser aplicado, como se muestra en la Tabla 2:

Tabla 2

Medida de Adecuación Muestral de Kaiser - Meyer - Olkin

KMO y prueba de Bartlett

\begin{tabular}{|c|c|c|}
\hline \multicolumn{2}{|c|}{ Medida de adecuación muestral de Kaiser-Meyer-Olkin. } & .938 \\
\hline \multirow{3}{*}{ Prueba de esfericidad de Bartlett } & Chi-cuadrado aproximado & 3634.299 \\
\hline & Gl & 253 \\
\hline & Sig. & .000 \\
\hline
\end{tabular}

(Elaboración propia, 2014).

2. De acuerdo a la Matriz de Componentes Rotados, a través de los Métodos de Extracción de Análisis de Componentes Principales y de Rotación de Normalización Varimax con Kaiser se obtuvieron tres categorías principales para 22 reactivos (eliminando uno de ellos), las cuales están en función a las variables del Modelo de Liderazgo Educativo: Liderazgo Pedagógico, Liderazgo Directivo y Liderazgo Institucional.

En la versión final del cuestionario, se consolidó con 22 reactivos auto - administrados con una escala de medición tipo Likert que es la que a continuación se expone:

1 Totalmente en desacuerdo

2 En desacuerdo

3 Ni de acuerdo ni en desacuerdo 
4 De acuerdo

5 Totalmente de acuerdo

Asimismo y en complementación con el Análisis Factorial Exploratorio, una vez reorganizados los reactivos, se realizó la Correlación de Pearson entre variables, para determinar su significancia indicando la existencia de variables con correlaciones entre sí elevadas y con significancia de cero, lo que comprueba que es un instrumento válido, ya que de acuerdo con Morales Vallejo (2013), una correlación es significativa a partir de .7.

Finalmente, en relación con la confiabilidad, ésta se obtuvo una vez validado el instrumento a través del Alfa de Cronbach, por medio del software estadístico SPSS 20.1, reportando los datos expuestos en la Tabla 3:

Tabla 3

Alfa de Cronbach del Instrumento

\begin{tabular}{cc}
\hline VARIABLE & ALFA DE CRONBACH \\
\hline Liderazgo Institucional & .937 \\
Liderazgo Directivo & .913 \\
Liderazgo Pedagógico & .855 \\
Alfa de Cronbach de todo el instrumento & .962 \\
\hline
\end{tabular}

(Elaboración propia, 2014)

En todos los casos la confiabilidad es mayor a .7 que de acuerdo con Morales Vallejo (2013), los valores mayores a .7 son aceptables para investigaciones exploratorias.

De lo anterior, se atestigua que el instrumento diseñado para la presente investigación es válido y confiable para ser aplicado.

\section{Procedimiento de recolección de datos (aplicación del instrumento)}

La recolección de datos para el desarrollo de la presente investigación se llevó a cabo por medio de la aplicación del cuestionario: "Liderazgo Educativo", siguiendo las actividades a continuación numeradas:

1. Identificación de las Instituciones de Educación Superior del Valle de Toluca.

2. Ubicación de los contactos pertinentes en cada Organismo Académico, para gestionar el permiso de aplicación de los cuestionarios.

3. Una vez dado el permiso, entrega del cuestionario a los participantes.

4. Auto-aplicación de los cuestionarios: los cuestionarios fueron entregados a los participantes quienes se quedaron con ellos aproximadamente un par de días.

5. Recuperación de los cuestionarios contestados.

6. Codificación computarizada de los datos.

7. Análisis y diagnóstico de resultados. 


\section{Análisis de resultados}

\section{Liderazgo Institucional}

De acuerdo a lo arrojado por el instrumento, la media de la variable Liderazgo Institucional es de 3.92 con una desviación típica de .559, lo que implica que la curva de datos se encuentra entre las puntuaciones: 3.36 y 4.47, indicando que la mayoría de los encuestados contestó estar "De acuerdo" con las prácticas realizadas para esta variable, no obstante, hubo un mínimo de participantes que también respondieron en las ponderaciones menores a 3 en función a una escala Likert de 5 puntos en donde 5, es la puntuación más favorable.

Referente a los resultados arrojados por la prueba Chi Cuadrado de Pearson, en la Tabla 4, se muestra que para el tipo de docente, ya sea docente de medio tiempo, tiempo completo $\mathrm{u}$ horas clase, sí existen diferencias estadísticamente significativas en los reactivos: 1) El equipo directivo constituye conocimientos y habilidades, 4) Evaluación y apoyo a otros centros, 5) Motivación y Compromiso, 10) Marco legal

Tabla 4

Chi cuadrado de Pearson, Liderazgo Institucional

\begin{tabular}{|c|c|c|c|c|c|c|}
\hline \multirow{2}{*}{$\begin{array}{c}\text { PRUEBA DE CHI } \\
\text { CUADRADO DE PEARSON }\end{array}$} & \multicolumn{3}{|c|}{ TIPO DE DOCENTE } & \multicolumn{3}{|c|}{$\begin{array}{l}\text { TIPO DE } \\
\text { INSTITUCIÓN }\end{array}$} \\
\hline & $\begin{array}{c}\text { Chi } \\
\text { cuadrado }\end{array}$ & gl & Sig. & $\begin{array}{c}\text { Chi } \\
\text { cuadrado }\end{array}$ & gl & Sig. \\
\hline $\begin{array}{l}\text { El equipo directivo construye el conocimiento y } \\
\text { habilidades }\end{array}$ & 7.471 & 4 & $.113^{a, b}$ & 12.400 & 4 & $.015^{\mathrm{a}, \mathrm{b}, *}$ \\
\hline Influencia en otros organismos & 21.166 & 3 & $.000^{\mathrm{a}, *}$ & 6.587 & 3 & $.086^{\mathrm{a}}$ \\
\hline $\begin{array}{l}\text { El dirigente da solución a los problemas de for- } \\
\text { ma creativa e innovadora }\end{array}$ & 21.574 & 4 & $.000^{\mathrm{a},{ }^{*}}$ & 8.628 & 4 & $.071^{\mathrm{a}}$ \\
\hline Evaluación y apoyo a otros centros & 14.150 & 4 & $.007^{\mathrm{a}, \mathrm{b}, *}$ & 5.916 & 4 & $.206^{\mathrm{a}, \mathrm{b}}$ \\
\hline Motivación y compromiso & 14.784 & 3 & $.002^{\mathrm{a}, *}$ & 7.539 & 3 & $.057^{\mathrm{a}}$ \\
\hline $\begin{array}{l}\text { El equipo directivo se adapta a los cambios y los } \\
\text { genera }\end{array}$ & 18.941 & 3 & $.000^{\mathrm{a}, *}$ & 22.579 & 3 & $.000^{\mathrm{a}, *}$ \\
\hline El director escolar es un referente como persona & 24.297 & 4 & $.000^{\mathrm{a}, *}$ & 10.971 & 4 & $.027^{\mathrm{a}, *}$ \\
\hline $\begin{array}{l}\text { El director escolar tiene buenas relaciones inter- } \\
\text { personales }\end{array}$ & 20.694 & 4 & $.000^{\mathrm{a}, \mathrm{b}, *}$ & 6.299 & 4 & $.178^{\mathrm{a}, \mathrm{b}}$ \\
\hline El director es un excelente comunicador & 29.216 & 4 & $.000^{\mathrm{a}, \mathrm{b},{ }^{*}}$ & 14.179 & 4 & $.007^{\mathrm{a}, \mathrm{b}, *}$ \\
\hline Marco legar institucional & 16.374 & 4 & $.003^{\mathrm{a}, \mathrm{b}, *}$ & 12.960 & 4 & $.011^{\mathrm{a}, \mathrm{b}, *}$ \\
\hline Distribución de tareas y obligaciones & 8.104 & 4 & $.088^{\mathrm{a}, \mathrm{b}}$ & 2.255 & 4 & $.689^{\mathrm{a}, \mathrm{b}}$ \\
\hline
\end{tabular}

Los resultados se basan en filas y columnas no vacías de cada subtabla más al interior.

*. El estadístico de chi-cuadrado es significativo en el nivel .05.

a. Más del 20\% de las casillas de esta subtabla esperaban frecuencias de casilla inferiores a 5. Puede que los resultados de chi-cuadrado no sean válidos.

b. Las frecuencias esperadas de casilla mínimas en esta subtabla son inferiores a uno. Puede que los resultados de chi-cuadrado no sean válidos. (Elaboración propia, 2014). 
institucional y 11) Distribución de tareas y obligaciones. Siendo los profesores de horas clase los que mejor evalúan estos aspectos, ya que en promedio el $79 \%$ de ellos manifiestan estar de acuerdo y en total acuerdo con estos aspectos. Por su parte, en promedio, el $12 \%$ de los docentes de medio tiempo y tiempo completo manifiestan estar en total acuerdo en estas prácticas y sólo un 59\% manifiesta estar de acuerdo.

Por último para esta variable, en cuanto al tipo de institución en donde laboran los docentes, sea esta pública o privada, sí se encuentran diferencias significativas para todos los reactivos exceptuando el reactivo número 6: El equipo directivo se adapta a los cambios y los genera. En esta variable fueron los docentes que laboran en instituciones públicas los que mejor evalúan estas prácticas de su líder ya que en promedio únicamente el 3\% de ellos manifestó estar en completo desacuerdo y en desacuerdo, mientras que en promedio el $75 \%$ de los mismos refirió estar de acuerdo y completamente de acuerdo con tales prácticas, en comparación con el $70 \%$, en promedio, de profesores que laboran en instituciones privadas, quienes manifiestan estar en completo acuerdo y en acuerdo con estos aspectos del Liderazgo Institucional.

\section{Liderazgo Directivo}

Para esta variable, la media estadística es 3.94 con una desviación típica de .611, lo que implica que la curva de datos se encuentra entre las puntuaciones 3.32 y 4.55, lo que indica que en su mayoría los encuestados respondieron estar de acuerdo con las actividades propias de esta variable.En virtud de lo anterior, se refiere que si bien en su mayoría hay encuestados que respondieron ponderaciones de satisfacción, también hubo, en menor porcentaje, quienes no están de acuerdo o están completamente en desacuerdo, para las actividades propias de esta variable.

Con referencia a la prueba Chi cuadrado de Pearson se observa que para las variables: Tipo de Docente y Tipo de Institución, sí existen diferencias estadísticamente significativas en relación a todos los ítems de la variable Liderazgo Directivo, reportando lo que se observa en la Tabla 5.

De lo anterior se obtiene que de acuerdo al tipo de docentes, el $68 \%$ en promedio, de los que trabajan medio tiempo y tiempo completo, refieren estar de acuerdo y totalmente de acuerdo con las prácticas de Liderazgo Directivo llevadas a cabo en su organización, mientras que el $27 \%$ de los mismos manifestó indiferencia. En comparación con los profesores que laboran por horas clase, dado que el $78 \%$ de ellos percibe que su líder gestiona adecuadamente el liderazgo directivo y únicamente un $4 \%$ refrió no estar de acuerdo o en completo desacuerdo con tales actividades.

Para la variable tipo de institución, se observa que el 20\% de los docentes que laboran en instituciones públicas manifestó indiferencia ante las actividades que su directivo realiza enfocados a Liderazgo Directivo, mientras que el 77\% de los mismos refirió estar de acuerdo y totalmente de acuerdo para estas prácticas. No así el 18\% de los profesores que laboran en instituciones particulares quienes mostraron indiferencia hacia sus líderes para esta variable, siendo el $70 \%$ de ellos quienes manifestaron conformidad y por el ende el $12 \%$ reportan no estar de acuerdo o en completo desacuerdo para esta variable. 
Tabla 5

Chi cuadrado de Pearson, Liderazgo Directivo

\begin{tabular}{|c|c|c|c|c|c|c|}
\hline \multirow{2}{*}{$\begin{array}{c}\text { PRUEBA DE CHI } \\
\text { CUADRADO DE PEARSON }\end{array}$} & \multicolumn{3}{|c|}{ TIPO DE DOCENTE } & \multicolumn{3}{|c|}{$\begin{array}{c}\text { TIPO } \\
\text { DE INSTITUCIÓN }\end{array}$} \\
\hline & $\begin{array}{c}\text { Chi } \\
\text { cuadrado }\end{array}$ & gl & Sig. & $\begin{array}{c}\text { Chi } \\
\text { cuadrado }\end{array}$ & Gl & Sig. \\
\hline Evaluación al trabajo realizado & 14.327 & 4 & $.006^{*}, \mathrm{~b}, \mathrm{c}$ & 13.395 & 4 & $.009^{*, \mathrm{~b}, \mathrm{c}}$ \\
\hline $\begin{array}{l}\text { Esfuerzo por generar estructuras partici- } \\
\text { pativas }\end{array}$ & 11.315 & 4 & $.023^{*}, \mathrm{~b}, \mathrm{c}$ & 15.794 & 4 & $.003^{*}, \mathrm{~b}, \mathrm{c}$ \\
\hline $\begin{array}{l}\text { El equipo directivo es visionario y piensa } \\
\text { estratégicamente }\end{array}$ & 13.161 & 4 & $.011^{*, b, c}$ & 9.597 & 4 & $.048^{*, \mathrm{~b}, \mathrm{c}}$ \\
\hline Clima Organizacional & 15.435 & 4 & $.004^{*, b, c}$ & 15.386 & 4 & $.004^{*, b, c}$ \\
\hline $\begin{array}{l}\text { El equipo directivo fomenta condiciones } \\
\text { de trabajo docentes }\end{array}$ & 10.009 & 4 & $.040^{*}, \mathrm{~b}, \mathrm{c}$ & 11.588 & 4 & $.021^{*, \mathrm{~b}, \mathrm{c}}$ \\
\hline $\begin{array}{l}\text { Gestión de prácticas asociadas en el salón } \\
\text { de clase }\end{array}$ & 15.199 & 4 & $.004^{*}, \mathrm{~b}$ & 6.639 & 4 & $.156^{\mathrm{b}, \mathrm{c}}$ \\
\hline
\end{tabular}

Los resultados se basan en filas y columnas no vacías de cada subtabla más al interior.

*. El estadístico de chi-cuadrado es significativo en el nivel .05.

b. Más del $20 \%$ de las casillas de esta subtabla esperaban frecuencias de casilla inferiores a 5. Puede que los resultados de chi-cuadrado no sean válidos.

c. Las frecuencias esperadas de casilla mínimas en esta subtabla son inferiores a uno. Puede que los resultados de chi-cuadrado no sean válidos.

(Elaboración propia, 2014)

\section{Liderazgo Pedagógico}

Finalmente, para la última de las variables independientes: Liderazgo Pedagógico, la estadística muestra que en una escala de 5 puntos (en donde 5 es la puntuación de mayor grado de aceptación), tiene una media de 3.95 con una desviación típica de .634, lo que indica que la curva se encuentra entre las puntuaciones 3.31 y 4.58 , encontrándose un ítem con una media de 4.02, siendo este el mejor calificado de todo el instrumento.

Complementando lo anterior y de lo arrojado por la prueba Chi cuadrado de Pearson, en la Tabla 6, se obtiene que para la variable tipo de docente, el único reactivo que no fue estadísticamente significativo para los profesores de tiempo completo y medio tiempo así como para los de horas clase fue el 22: Dentro de la organización el jefe o autoridad señala lo que se debe realizar, dado que todos los demás muestran significancia estadística en sus respuestas, en donde únicamente el $64 \%$ de los docentes que laboran tiempo completo y medio tiempo manifestaron estar de acuerdo y completamente de acuerdo para las prácticas que su directivo hace del Liderazgo Pedagógico, siendo el $34 \%$, en promedio, quienes mostraron indiferencia y por lo tanto el $2 \%$ manifestó estar en desacuerdo o totalmente en desacuerdo. En contraste, el $77 \%$ de los profesores que trabajan por horas clase manifestó estar en acuerdo o completamente de acuerdo para 
los ítems de esta variable, mientras que el 18\% se mostró indeciso al contestar no estar en desacuerdo pero tampoco de acuerdo con lo realizado en su institución en cuanto al Liderazgo Pedagógico.

Finalmente, en cuanto al tipo de institución, todos los reactivos tuvieron significancia estadística, lo que indica que sí existen diferencias en cuanto a la gestión del Liderazgo Pedagógico en las instituciones públicas y privadas; ya que en promedio el $23 \%$ de los profesores que laboran en intuiciones públicas mostraron indiferencia en cuando a la gestión de estas actividades, mientras que el 73\% de ellos muestra estar en acuerdo o totalmente de acuerdo. No obstante, el 10\% de los profesores que se desempeñan en instituciones particulares refieren indecisión al manifestar no estar de acuerdo ni en desacuerdo con lo que se hace en sus organismos para estas actividades y el 83\%, refieren estar de acuerdo y completamente de acuerdo, lo que indica que los profesores que laboran en instituciones privas evalúan mejor a sus directivos para la variable Liderazgo Pedagógico.

Tabla 6

Chi cuadrado de Pearson, Liderazgo Pedagógico

\begin{tabular}{|c|c|c|c|c|c|c|}
\hline \multirow{2}{*}{$\begin{array}{c}\text { PRUEBA DE CHI } \\
\text { CUADRADO DE PEARSON }\end{array}$} & \multicolumn{3}{|c|}{ TIPO DE DOCENTE } & \multicolumn{3}{|c|}{$\begin{array}{l}\text { TIPO DE } \\
\text { INSTITUCIÓN }\end{array}$} \\
\hline & $\begin{array}{c}\text { Chi } \\
\text { cuadrado }\end{array}$ & gl & Sig. & $\begin{array}{c}\text { Chi } \\
\text { cuadrado }\end{array}$ & gl & Sig. \\
\hline $\begin{array}{l}\text { Preocupación por los logros académicos de } \\
\text { la institución. }\end{array}$ & 9.498 & 4 & $.050^{*}, \mathrm{~b}$ & 7.763 & 4 & $.101^{b, c}$ \\
\hline Fomento del trabajo en equipo. & 19.855 & 4 & $.001^{*}, \mathrm{~b}$ & 2.321 & 4 & $.677^{\mathrm{b}}$ \\
\hline $\begin{array}{l}\text { Preocupación por el proceso de enseñanza - } \\
\text { aprendizaje. }\end{array}$ & 19.359 & 4 & $.001^{*, \mathrm{~b}, \mathrm{c}}$ & 7.495 & 4 & $.112^{b, c}$ \\
\hline $\begin{array}{l}\text { Los resultados de uno afectan lo que realiza } \\
\text { otro. }\end{array}$ & 13.108 & 4 & $.011^{*, b, c}$ & 15.770 & 4 & $.003^{*, b, c}$ \\
\hline $\begin{array}{l}\text { Dentro de la organización el jefe o autoridad } \\
\text { señala lo que se debe realizar. }\end{array}$ & 22.452 & 4 & $.000^{*}, \mathrm{~b}$ & 7.132 & 4 & $.129^{b}$ \\
\hline
\end{tabular}

Los resultados se basan en filas y columnas no vacías de cada subtabla más al interior.

*. El estadístico de chi-cuadrado es significativo en el nivel .05.

b. Más del $20 \%$ de las casillas de esta subtabla esperaban frecuencias de casilla inferiores a 5 . Puede que los resultados de chi-cuadrado no sean válidos.

c. Las frecuencias esperadas de casilla mínimas en esta subtabla son inferiores a uno. Puede que los resultados de chi-cuadrado no sean válidos.

(Elaboración propia, 2014)

\section{Discusión y conclusiones}

En los últimos diez años, el estudio científico del ámbito educativo, en el área específica del recurso humano, se ha incrementado notablemente debido a las condiciones sociales tan cambiantes en las que se vive. Competitividad ya no es un 
término meramente utilizado en el sector industrial o empresarial, ahora, no sólo se exigen organizaciones competitivas, sino que además los individuos que laboran en esas organizaciones deben ser altamente competitivos y poseer un capital intelectual sólido que permita desarrollar innovación continua y vanguardista para ubicar a sus empresas dentro de las mejores a nivel global. De ahí el trabajo fundamental que tienen con la sociedad las Instituciones de Educación Superior, ya que su premisa básica implica formar personal cualificado y calificado para desarrollarse en la industria tan exigente del Siglo XXI.

En virtud de lo anterior, para formar personas con conocimientos integrales y de alta calidad, se requiere que las Instituciones de Educación Superior, se gestionen, en todos sus niveles, por líderes capaces de atender tanto a las necesidades sociales de su entorno, como las de sus trabajadores, para que así contribuyan al fortalecimiento del sector educativo superior. Una institución educativa de nivel superior que sea dirigida por un equipo directivo que tenga únicamente conocimientos técnicos en un ramo de su oferta de estudios, perderá una parte importante de su administración educativa, ya que no se tendrá una visión global de lo que se necesita para la gestión escolar integral. Por otro lado, si el equipo directivo son, más que directores escolares, líderes educativos, el proceso de gestión escolar retoma otra línea de acción dirigida a ser Instituciones de Educación Superior competentes y de calidad, ya que la visión que tiene un líder es más amplia y estratégica que la de un director.

Un líder educativo debe, como primera instancia, tener los conocimientos fundamentales en cuestiones administrativas que sustenten su gestión adecuada, mirando a la escuela como una organización que requiere una proyección institucional pertinente. En segunda instancia, pero no menos importante, los líderes educativos de las Instituciones de Educación Superior, tienen que desarrollar la capacidad de planeación y gestión administrativa escolar que es característica propia de los directivos en el sentido estricto. Finalmente, un líder educativo, debe sustentar su práctica en el ser o estar de su organización, es decir, en la meta de toda Institución de Estudios Superiores: la educación integral de su alumnado, de ello la importancia de un liderazgo que vaya más allá de las prácticas meramente escolares, y se concentre en todo el proceso pedagógico educativo.

En lo preliminar recae la pertinencia e importancia de la presente investigación, en donde se propone que las comunidades escolares - y sus actores - de las IES del Valle de Toluca sean gestionadas por medio de un modelo de liderazgo integral que abarque los tres factores anteriormente mencionados: el Liderazgo Institucional, el Liderazgo Directivo y el Liderazgo Pedagógico, de los cuales se desprende el Modelo de Liderazgo Educativo, el cual se tomó como el referente principal para el desarrollo de la misma.

Focalizando el proyecto al fomento del Modelo del Liderazgo Educativo en las Instituciones de Educación Superior del Valle de Toluca, se dieron respuestas a las preguntas de investigación planteadas lográndose los objetivos propuestos.

En función a lo anterior, también es importarte mencionar que el seguimiento y aplicación de las variables del Modelo de Liderazgo Educativo: Liderazgo Institucional, Liderazgo Directivo y Liderazgo Pedagógico, no limita a los líderes educativos a no poder utilizar características de otros tipos de liderazgo, de hecho se sugiere que así sea. 
En cuanto al alcance del proyecto, se sugiere que futuras investigaciones se focalicen en implementar, aplicar, desarrollar, evaluar, mejorar e innovar estrategias para que se dé un cambio dinámico y flexible, ya que para el fortalecimiento total del Modelo de Liderazgo Educativo se deben generar mejoras continuas que: 1) se adapten a las necesidades específicas de cada centro escolar y 2) se adapten a los cambios tan demandantes en el sector educativo que se exigen a nivel local, estatal, nacional e incluso internacional.

En correlación con lo anterior, la investigación presentada únicamente para las Instituciones de Educación Superior del Valle de Toluca, puede servir como base para desarrollar futuras investigaciones en regiones diferentes a nivel local, estatal y nacional, para complementar el estudio presentado y generar una visión global de la situación actual del liderazgo educativo en las IES de otras localidades. Asimismo, se sugiere que también se desarrollen líneas de investigación similares en sectores educativos de otros niveles: nivel medio superior y nivel básico, para que de esta forma, se desarrollen planes de acción que fomenten el Liderazgo Educativo en estos sectores.

Por otro lado, en cuanto a las dificultades presentadas para el desarrollo de la investigación, se encontraron aquellas de índole técnico, como el cumplimiento formal de la representatividad de la muestra, que si bien no tuvo implicaciones cruciales en el cumplimiento de los objetivos de investigación, en su momento si fue un problema menor.

Finalmente, a manera de conclusión, se resume que el liderazgo es un sine qua non que fortalece el área de Recursos Humanos a nivel industrial y empresarial. Así, en el ámbito universitario, el Liderazgo Educativo, a través de sus tres variables: Liderazgo Institucional, Liderazgo Directivo y Liderazgo Pedagógico, permite la administración adecuada de las instituciones involucrando a todos los actores de la comunidad escolar. Dentro de los estudios superiores, la gestión pertinente y efectiva del Liderazgo Educativo admite atender tanto las necesidades de una sociedad altamente dinámica en el cambio de conocimientos, que exige la formación de individuos más competitivos; como las necesidades de docentes y administrativos que en ellas laboran y que demandan líderes integrales que sean capaces de atender todas las directrices en las que versa la educación superior actual.

\section{Bibliografía}

Abad, J. de V. (2010). 7 ideas clave. Escuelas sostenibles en convivencia. España: Graó.

Adan, E. (2006). Liderazgo renovador y esperanzador para construir la universidad del futuro. Laurus, 12(Ext), 170-179. Recuperado de http://www.redalyc.org/articulo. oa?id=76109910

Ahumada, L. (2012). Liderazgo en organizaciones educativas. Persona, 15, $239-252$. Recuperado de http://www.redalyc.org/articulo.oa?id=147125259014

Álvarez Botello, J., Chaparro Salinas, E. M., Ruíz Tapia, J. A., Romero Romero, A., Heredia Dominico, R. y Martínez Ávila, M. (2012). Administración educativa. Un reto para la competitividad y la pertinencia. México: Universidad Autónoma del Estado de México.

Asociación Nacional de Universidades e Instituciones de Educación Superior. (ANUIES) (2012). Anuario estadístico de educación superior. Recuperado de: http://www. anuies.mx/content.php?varSectionID $=166$ 
Cheong Cheng, Y. (2011). Towards the 3rd wave school leadership. Revista de Investigación Educativa, 29(2), 253-275. Recuperado de: http://revistas.um.es/rie/article/ view/130811

Elizondo Montemayor, L. (2011). Competencias que debe tener un director académico universitario para la educación superior basada en competencia. Revista de Investigación Educativa, 29(1), 205-218. Recuperado de: http://revistas.um.es/rie/article/ view/110791/127042

Garbanzo, G. y Orozco, V. (2010). Liderazgo para una gestión moderna de procesos educativos. Educación, 34(1), 15-29. Recuperado de http://www.redalyc.org/articulo. oa? id=44013961001

González González, O. (2008). Liderazgo transformacional en el docente universitario. Maracaibo, Venezuela. Universidad del Zulia. Multiciencias, 8(1), 38-47. Recuperado de http://www.redalyc.org/articulo.oa?id=90480105

González González, O. y Gónzalez Cubillan, L. (2012). Estilos de liderazgo del docente universitario. Multiciencias, 12(1), 35-44. Recuperado de http://www.redalyc.org/ articulo.oa?id=112345259014

Hernández Sampiere, R., Fernández Collado, C. y Baptista Lucio, P. (5 $5^{\mathrm{a}}$ ed.). (2010). Metodología de la Investigación. México: McGraw Hill.

López Leiva, V. (2010). Liderazgo y mejora educativa. Psicoperspectivas, 9(2), 1-8. Recuperado de: http://www.redalyc.org/articulo.oa?id=11234524

Lussier, R. N. y Achua, C. F. (4-⿳⺈⿴囗十一 ed.). (2010). Liderazgo. Teorías, aplicación y desarrollo de habilidades. México: CengageLearning.

Martín Martín, Q., Cabero Morán, M. A. y De Paz Santana Y. del R. (2008). Tratamiento estadístico con datos SPSS. Practicas resueltas y comentadas. Madrid, España: Thomson.

Morales Vallejo, P. (2013). El análisis factorial en la construcción e interpretación de tests, escalas y cuestionarios. Universidad Pontifica Comillas: Madrid. Recuperado de: http://web.upcomillas.es/personal/peter/investigacion/AnalisisFactorial.pdf

Rodríguez - Molina, G. (2011). Funciones y rasgos del liderazgo pedagógico en los centros de enseñanza. Educación y Educadores, 12(2), 253-267. Recuperado de http:// www.redalyc.org/articulo.oa?id=83421304010

Sánchez, V., Tapia, C. y Becerra, S. (2006). Liderazgo: una mirada desde directivos, profesores y alumnos. Revista de Psicología, XV(2), 29-52. Recuperado de http://www. redalyc.org/articulo.oa?id=26415202

SummerDonna, C. S. (2006). Administración de la Calidad. México: Pearson Educación. Tapia, C., Mansilla-Sepúlveda, J., Becerra, S. y Saavedra, J. (2011). Liderazgo de los directivos docentes en contextos vulnerables. Educación y Educadores, 14(2), 389-409. Recuperado de http://www.redalyc.org/articulo.oa?id=83421404010

Fecha de recepción: 24/09/2014

Fecha de revisión: 26/09/2014

Fecha de aceptación: 25/05/2015 